\title{
Perspective management of peripartum cardiomyopathy case (PPCMP)
}

\begin{abstract}
Peripartum Cardiomyopathy is a rare disease presenting with acute heart failure symptoms during the last month of pregnancy up to 5th month after delivery. PPCMP carries significantly high mortality and morbidity threatening the life of mother and fetus. Therefore, timely diagnosis of the disease is very crucial to the management of patient and in improving the prognosis. These study overviews briefly about the presentation, diagnosis and practical ethical aspects of the disease management.
\end{abstract}

Volume 3 Issue 3 - 2015

\author{
Haseeb Ashraf \\ King Edward Medical University, Pakistan
}

Correspondence: Haseeb Ashraf, King Edward Medical University, Pakistan Registrar Cardiac ICU at AI-Dar Hospital, Madina Munawwara, Neela Gumbad, Anarkali, Lahore, Pakistan, Tel 923219200037, Email styledmussel@hotmail.com

\section{History}

Thirty three yearsold, female patient with 36 weeks of gestation presented with shortness of breath (SOB) for the last 2 weeks. The condition started by dyspnea during 2 nd trimester which is mild in exertions, rapidly progressive till reach duringher daily activities last 2 weeks and associated with paroxysmal nocturnal dyspnea. She also noted edema in her feet. She does not have any other systemic complaints. No past history of chronic diseases, operation or similar attack, however, she recalls that her elder sister once had similar complaints during her last pregnancy which ended up in her death. She has 2 kids who are healthy. Her previous pregnancies went unremarkable.

\section{Examination}

On examination: blood pressure was $116 / 66 \mathrm{mmHg}$, Pulse 98/ min, and normal temperature and $\mathrm{O} 2$ saturation. Cardiac auscultation revealed loud S1, normal S2 and an ejection systolic murmur at the left 2 nd intercostal space and another systolic murmur at apex. ${ }^{1,2}$ Also fine inspiratory basal crepitation was detected on auscultation of bothlungs. No evidence of organomegaly or ascites however, pitting edema on both feet up to the mid of lower legs was observed. Neurological examination was normal.

\section{Diagnosis/Differential diagnosis}
a. Cardiomyopathy.
b. Pulmonary Embolism.
c. Valvular heart disease complicated with heart failure.
d. Lower respiratory tract infection.
e. Acute Coronary syndrome.

\section{Investigations and intervention}
a. Hemoglobin $=11.2 \mathrm{~g} / \mathrm{dl}$
b. Platelets $=250,000$
c. $\mathrm{WBCs}=8000$
d. LFTs $=$ Normal
e. $\operatorname{Urea}=40$
f. Creatinine $=1.1 \mathrm{mg} / \mathrm{dl}$

g. Urine Complete $=$ Normal

h. BNP levels = significantly raised

i. ECG show normal sinus rhythm at rate of $98 / \mathrm{min}$, Poor progression of $\mathrm{R}$ waves with $\mathrm{T}$-wave inversion involving anterior precordial leads.

j. $\quad$ CXR $=$ Shows picture of pulmonary edema with kerley B lines at bases and upper lobe diversion of the prominent vascular markings.

k. Echocardiography = globally hypokinetic, dilated left ventricular cavity with moderately severe LV systolic dysfunction (EF 32\%). Diastolic measurements show Grade III dysfunction. CFM shows moderate MR. No evidence of pericardial effusion. No evidence of intracavitarythrombus.

1. Her fetal assessment showed a live fetus with normal fetal heart activity and growth.

\section{Treatment}

Patient was treated under lines of Heart failure except for Ace inhibitors. She responded very well to following medications

a. Intermittent Supplemental Oxygen by nasal canula.

b. Upright posture maintenance.

c. Diuresis with Intravenous furosemide at $40 \mathrm{mg}$ twice daily.

d. Beta blockade with Carvedilol $3.125 \mathrm{mg}$ twice daily. ${ }^{3,4}$

e. Tab Digoxin $0.125 \mathrm{mg}$ once daily.

f. She was planned for Normal Vaginal Delivery as the baby was alive and healthy.

\section{Discussion}

Peripartum Cardiomyopathy (PPCMP) is defined by the European society of cardiology (ESC) as "the idiopathic cardiomyopathy presenting in heart failure symptoms resulting in left ventricular (LV) systolic dysfunction $(<45 \%$ EF) at the last month of pregnancy up to 5 th month post partum and LV dysfunction should not have any other defined reason". According to the definition, three criteria should be met;

i. Heart failure developing between the last month of pregnancy to 5 th month after delivery. 
ii. LV systolic dysfunction resulting in reduced ejection fraction $(<45 \%)$ with or without LV cavity dilatation.

iii. There should be no other reason for the reduced ejection fraction.

PPCMP has to be a diagnosis of exclusion after investigating vigorously for other causes of heart failure. Etiology and pathogenesis of PPCMP is not known. However, many hypotheses has been postulated derived from different researches blaming the oxidative stress, autoimmune responses, genetic factors, Nutritional factors, Viral syndromes and inflammatory pathways to be involved. One of such theories is a Prolactin induced STAT3 pathway activation. ${ }^{5,6}$

Diagnosing PPCMP poses difficulty in a way that it presents in the late pregnancy by which the symptoms are masked or overlooked. The sooner the diagnosis is suspected the sooner it is referred and managed. For ease, Fett and colleagues have suggested a probability score for PPCMP (Table 1). 0-2 Low Risk Observation.

a. 3-4 Moderate Risk Consider BNP.

b. $>=5$ High RisksConsider BNP and Echocardiography.

Table I This table has been taken from a ESC presentation slide

\begin{tabular}{ll}
\hline Detet rminan & Persistent LV Dysfunction \\
\hline Symptoms of Heart Failure & $50 \%$ \\
Decrease in LVEF > 20\% & $33 \%$ \\
LVEF Recovery & $60 \%$ \\
Death & $25 \%$ \\
Premature Delivery & $50 \%$ \\
Therapeutic Abortion & $25 \%$ \\
\hline
\end{tabular}

Identification of high risk patients before hand is deemed necessary in order to treat them aggressively and under intensive care. The high risk features are;

1. Ejection fraction $<30 \%$

2. Requiring Inotropic support

3. Intolerant to Betablockers

\section{Management of PPCMP}

Acute Setting before Delivery In the acute setting it is necessary to triage the patient whether or not intensive care monitoring is required. Patients should be managed on the line of Acute heart failure which have shown to improve symptoms and prognosis in otherwise primary cardiomyopathy.

a. If inotropic support is required patient should be considered for dobutamine infusion or digoxin therapy. And if critically ill then invasive hemodynamic support with intra-aortic balloon pump (IABP) could be required.

b. Diuretics like furosemide should be considered in patient with pulmonary edema andorthopneato relive of their respiratory distress and alleviate pulmonary edema. Before intensively diuresis, diagnosis of preeclampsia is ruled out in which the patients are already volume depleted.

c. Patient should be given very low dose beta blockers.

d. Heparin if ejection fraction is less than $35 \%$.

e. ACE INHIBITORS AND ARBs ARE ABSOLUTELY CONTRAINDICATED.

f. Mode of Delivery should be VAGINAL until and unless required otherwise.
After the delivery has been carried out successfully, the treatment should be as follows

a. Inotropic support as required.

b. Beta blockers.

c. Ace Inhibitors or ARBs.

d. Diuretics (Loop or in combination with thiazide).

e. Hydralazine and Nitrates if hemodynamics allow and symptoms persist.

\section{f. Anticoagulation with Warfarin.}

There is a minimal data available in favor of bromocriptine therapy. One of the studies have shown that giving bromocriptine, $2.5 \mathrm{mg}$ twice daily for one week and then $2.5 \mathrm{mg}$ once daily for 6 weeks, improves prognosis significantly in terms of mortality and combined end points as compared to standard treatment. Counseling and patient education is one of the important aspects in the management of patient with PPCMP. As the risk of mortality and morbidity in subsequent pregnancies is way too high, pregnancy should be discouraged. If at all the patient conceives, she should be diligently and vigilantly managed. She should be put on Beta blockers and Ace inhibitors if she is already taking should be discontinued. She should be followed up with Echocardiography in each trimester and for 1, 3 and 6months after delivery. Risk of morbidity and mortality in subsequent pregnancy with persistent LV dysfunction is detailed in the table below. Practical Issues Concerning the Diagnosis and Management of PPCMP. When should the Dyspnea in pregnancy be evaluated? Normal Physiological changes alter the hemodynamics in such a way that the plasma volume increases by $50 \%$, Stroke volume, Cardiac Output and heart rate increases. Tidal volume, minute ventilation increases keeping the respiratory rate near normal. Increasing abdominal girth and pressure posed on the Inferior vena cava adds to the dyspnea. Therefore, dyspnea up to NYHA class II is a normal physiological phenomenon. Any dyspnea encroaching NYHA Class III-IV or with associated alarming features should be promptly investigated.

Pregnancy is a condition of hyperdynamic circulation, therefore, if the patient already has an underlying Cardiac failure, her failure may progress to high output failure. If she has an underlying silent valvular lesion, it may unmask with overwhelming dyspnea inconsistent to be explained with physiologic changes of pregnancy. One should not be worried about the ejection flow murmur at the 2 nd left intercostals space which is present in $98 \%$ of patients in pregnancy and it does not aggravate with inspiration. A continuous soft murmur of 'MammarySoufflés' may be heard. However, any diastolic murmur detected should be evaluated. What is the risk of Teratogenicity on exposure to X-rays?. Normal accepted value of X-rays exposure to fetus is up to 5 rads. Single X-ray exposure with protected shield on exposes to 0.00007 rads which means in others words is that she has been exposed to ONE X-ray out of 70,000 X-rays which are acceptable and safe for her fetus. This would be necessary to ensure the patient of the safety. Furthermore, a PA film lessens the X-ray exposure as compared to an AP film. If the patient is being suspected for coronary artery disease induced heart failure for she needs to be put under nuclear testing (Stress Thallium scan), she can safely undergo a stress thallium test which exposes to 0.1 rads (out of acceptable 5 rads) to fetus. This means that she has been exposed to 1 nuclear imaging test out of 50 which she can undergo safely without harming the fetus. Potential Side Effects of Over Exposure

\section{i. $5-10$ rads before 2 weeks $=$ Prenatal death .}



ii. 5-10 rads from 1-8 weeks $=$ Growth retardation.
iii. $>10$ rads from $8-25$ weeks $=$ Mental retardation .
iv. $>100$ rads after 25 weeks $=$ Still birth and Neonatal death.

\section{Mode of delivery}

Mode of delivery in PPCMP should be kept Vaginal except deemed necessary for Cesarean section. Because C-section can pose the risk of endometritis, pulmonary embolism, third spacing and even auto-transfusion causing an abrupt increase in the volume and thereby decompensate the failing heart. If the patient is made stable and fetus is healthy, labor could be induced with oxytocin and for pain relief, epidural or spinal block could be considered. Patient is not made to stress however, vacuum suction could be attempted in the third stage of labor if required. If there is a threat to the life of mother, cesarean section must be performed without any delay.

\section{Learning Points}

1. Diagnosis of PPCMP should be made after having ruled out other possible causes of dyspnea.

2. There should be no delay in making the diagnosis because of its high mortality.

3. Future pregnancy is absolutely contraindicated.

4. Multidisciplinary approach should be carried out in order to improve the patient and fetal outcome.

5. There is no recommendation as yet for Bromocriptine therapy, but however, if decided on individual basis, doses should be considered matching the pilot study.

\section{Acknowledgments}

None.

\section{Conflicts of interest}

Author declares there are no conflicts of interest.

\section{Funding}

None.

\section{References}

1. Elkayam U, Akhter MW, Singh H, et al. Pregnancy-associated cardiomyopathy: clinical characteristics and a comparison between early and late presentation. Circulation. 2005;111(16):2050-2055.

2. Ansari AA, Fett JD, Carraway RE, et al. Autoimmune mechanisms as the basis for human peripartum cardiomyopathy. Clin Rev Allergy Immunol. 2002;23(3):301-324.

3. Hilfiker-Kleiner D, Sliwa K, Drexler H. Peripartum cardiomyopathy: recent insights in its pathophysiology. Trends Cardiovasc Med. 2008;18(5):173-179.

4. Sliwa K, Blauwet L, Tibazarwa K, et al. Evaluation of bromocriptine in the treatment of acute severe peripartum cardiomyopathy: a proof-ofconcept pilot study. Circulation. 2010;121(13):1465-1473.

5. Fett JD, Fristoe KL, Welsh SN. Risk of heart failure relapse in subsequent pregnancy among peripartum cardiomyopathy mothers. Int $J$ Gynaecol Obstet . 2010;109(1):34-36.

6. Sliwa K, Hilfiker-Kleiner D, Petrie M C, et al. Current state of knowledge on aetiology, diagnosis, management, and therapy of peripartum cardiomyopathy: a position statement from the Heart Failure Association of the European Society of Cardiology Working Group on peripartum cardiomyopathy. European journal of heart failure. 2010;12(8):767-778.

7. Fett J. Crit pathways in cardiology. 2010. 